\title{
RESEARCH
}

\section{Prevalence of lower-extremity amputation among patients with diabetes mellitus: Is height a factor?}

\section{Chin-Hsiao Tseng}

An abridged version of this article appeared in the Jan. 3I, 2006, issue of CMAJ.

\section{ABSTRACT}

Background: Taller diabetic patients are at higher risk of peripheral sensory loss than shorter diabetic patients and thus may be at increased risk of lower-extremity ulcers and amputation. In a large telephone survey, the prevalence of lowerextremity amputation among patients with diabetes mellitus was determined and the association between height and lower-extremity amputation evaluated.

Methods: Of 256036 patients identified from hospital and clinic databases who had a diagnosis of diabetes and were seen at those institutions between 1995 and 1998, 128572 were randomly selected to be interviewed by telephone between 1995 and 2002. Of the 93484 patients who agreed to be interviewed, 386 were excluded (age $<18$ years); this left 93116 diabetec patients (42 970 men and 50146 women) for inclusion in the study.

Results: Of the 93116 patients interviewed, 3259 (3.5\%) had type 1 diabetes. Lower-extremity amputation was performed in $1.7 \%$ and $0.8 \%$ of the patients with type 1 and type 2 diabetes, respectively. The prevalence of amputation did not differ significantly between men and women with type 1 diabetes but was significantly higher among men than among women with type 2 diabetes (0.9\% v. $0.7 \%$ ). Height (every $10-\mathrm{cm}$ increment) was significantly associated with lowerextremity amputation (adjusted odds ratio [OR] 1.16, 95\% confidence interval $[\mathrm{Cl}]$ 1.03-1.32). In a subgroup of 9295 patients for whom data on fasting plasma glucose levels and dyslipidemia were available, and after additional adjustment for these 2 variables, body height remained an independent predictor of lower-extremity amputation (adjusted OR for every $10 \mathrm{~cm}$ of height $1.79,95 \% \mathrm{Cl}_{1.14}-2.82$ ).

Interpretation: Height is an independent predictor of lowerextremity amputation among patients with type 1 and type 2 diabetes mellitus.

Cite this article as $C M A J$ 2006;174(3). DOI:I0.1503/cmaj.050680

aller patients with diabetes mellitus have been shown to be more susceptible than shorter diabetic patients to peripheral sensory loss. ${ }^{1-7}$ This greater susceptibility could be due to increased vulnerability of longer nerve fibres to damage from external trauma or from the micro- or macrovascular complications of diabetes. Because peripheral sensory loss with the development of foot ulcers is the most common indication for lower-extremity amputation among diabetic patients, ${ }^{8}$ taller patients may be at increased risk of such a procedure. In Taiwan, although diabetes mellitus accounts for a third ${ }^{9}$ to half ${ }^{10}$ of all lowerextremity amputations, the procedure is much less common among Taiwanese people of shorter stature than among white people who are much taller. ${ }^{10}$ Similarly low procedure rates have been observed in other Asian populations. ${ }^{10}$ The reason for the difference in rates between ethnic groups is unknown, but height may be a factor.

The objectives of this study were to determine the prevalence of lower-extremity amputation among patients with diabetes mellitus (type I and type 2) and to evaluate the association between height and lower-extremity amputation, with adjustment for the potential confounding effects of age, sex, duration of diabetes, smoking status, hypertension, fasting plasma glucose level and dyslipidemia.

\section{Methods}

The study design was approved and supported by an ad hoc ethics committee of the Department of Health of Taiwan.

Because more than $96 \%$ of Taiwan residents (except for those who are involved in military service or are incarcerated) are covered by the compulsory National Health Insurance (NHI) plan, introduced in March 1995, and because the copayment rate is low ( $5 \%-30 \%$ for inpatient care and a fixed charge of about US\$I.40-\$12.4 for outpatient care), almost all diabetic patients are seen by NHI physicians. ${ }^{11,12}$ Taiwan is a small island, and hospitals are easily accessible by people in all parts of the island. Because patients do not need a referral to visit a doctor in a hospital and because hospitals provide better facilities and services than primary care physicians, most diabetic patients in Taiwan are cared for at hospitals. On average, a patient with diabetes visits an outpatient clinic 35.8 times a year. ${ }^{13}$ Reasons for these visits include getting prescriptions, dental care, eye examinations, physiotherapy, chiropractic care, acupuncture, and other medical consultations and procedures.

The databases of 66 hospitals and clinics located in every region of Taiwan were used to identify all patients seen in these institutions between I995 and I998 with a diagnosis of diabetes mellitus. The sample is described in detail else- 
Table 1: Baseline characteristics of patients with diabetes mellitus who participated in the survey

\begin{tabular}{|c|c|c|c|}
\hline Characteristic & $\begin{array}{c}\text { All patients } \\
n=93116\end{array}$ & $\begin{array}{l}\text { Patients with } \\
\text { type } 1 \text { diabetes } \\
n=3259\end{array}$ & $\begin{array}{l}\text { Patients with } \\
\text { type } 2 \text { diabetes } \\
n=89857\end{array}$ \\
\hline Age, mean (SD), yr & $62.0(11.6)$ & $56.2(16.6)$ & $62.2(11.3)$ \\
\hline Sex, male, \% (no.) & $46.1(42970)$ & $48.2(1572)$ & $46.1(41398)$ \\
\hline $\begin{array}{l}\text { Duration of diabetes, } \\
\text { mean (SD), yr }\end{array}$ & $7.3(6.6)$ & $10.3(7.9)$ & $7.2(6.5)$ \\
\hline Body height, mean (SD), cm & $160.1(7.9)$ & $161.1(8.2)$ & 160.1 (7.9) \\
\hline \multicolumn{4}{|l|}{ Smoking status, \% (no.) } \\
\hline Ever smoked & 30.7 (28 599) & $33.6(1095)$ & $30.6(27504)$ \\
\hline Never smoked & $69.3(64517)$ & $66.4(2$ 164) & $69.4(62353)$ \\
\hline Ex-smoker & $10.9(10123)$ & $8.6 \quad(280)$ & $11.0 \quad(9843)$ \\
\hline Current smoker & $19.8(18476)$ & $25.0 \quad(815)$ & 19.7 (17 661) \\
\hline Hypertension, \% (no.) & 54.2 (49 287) & $46.8(1478)$ & 54.5 (47 809) \\
\hline $\begin{array}{l}\text { Lower-extremity amputation, } \\
\% \text { (no.) }\end{array}$ & $0.8 \quad(784)$ & (54) & $(730)$ \\
\hline
\end{tabular}

Note: $\mathrm{SD}=$ standard deviation .

where. ${ }^{11,12}$ Of the 256036 patients identified, a sample of I28 572 was randomly chosen, with the expectation that $70 \%$ would complete the telephone interviews.

Between Mar. I, I995, and Apr. 30, 2002, trained interviewers administered the survey by telephone using a structured questionnaire; they made up to 3 attempts to reach subjects before excluding them from the study. To obtain as much information as possible and to keep missing data to a minimum, the interviewers handed the questionnaires in every week, and all returned questionnaires were checked for internal consistency by an assistant and then checked again by me. For questionnaires that had excessive amounts of missing data, the interviewers were asked to go back to the patients for more information.

The information obtained during the interview included demographic data; self-reported height and weight; ethnic background of both parents (respondents were asked to choose from 5 categories [Fukien, Hakka, Mainlander, Aborigine or other]); history of lower-extremity amputation; initial symptoms or clinical events that led to the diagnosis of diabetes; current treatment of diabetes; history of smoking; hypertension; family history of diabetes and hypertension; and use of betel nut (to evaluate health problems associated with betel-nut chewing).

A subset of the study population who reported having visited an NHI physician in the month before the interview were asked whether they had dyslipidemia and, if so, whether they had received treatment for it; they were also asked for the results of any fasting plasma glucose level obtained in the 3 months before the interview (the longest duration for which insulin and other drugs can be prescribed within the NHI).

Lower-extremity amputation was defined by a history of surgical resection of a part of the lower extremity on either side. Trauma-related amputations were not included. Type I diabetes was defined on the basis of one of the following criteria: diabetic ketoacidosis at the onset of diabetes mellitus, or the need for insulin injection within I year after the diagnosis of diabetes. If the patient did not have type I diabetes, he or she was considered to have type 2 diabetes. Hypertension was defined by a self-reported history and by a reported systolic blood pressure of $140 \mathrm{~mm} \mathrm{Hg}$ or greater, a diastolic pressure of $90 \mathrm{~mm} \mathrm{Hg}$ or greater, or both. For smoking status, patients were classified as never smokers, ex-smokers or current smokers.

Another interview, by someone other than the initial interviewer, was completed with 728 patients from a random sample of Iooo patients within 12 months after the first interview. Of the patients who were interviewed twice, 7 were consistent in reporting a lower-extremity amputation at both interviews; I patient reported undergoing the procedure between the first and second interviews. Inter-interviewer reliability was determined by correlation coefficients for the continuous variables (age at onset of diabetes, height, and systolic and diastolic blood pressures) and by percent agreement for the categorical variables (smoking status and hypertension). The correlation coefficients for the 2 interviewers for the continuous variables were $0.972,0.904,0.626$ and 0.436 , respectively $(p<0.0$ I for all); the percent agreement for the categorical variables was $91.7 \%$ and $97.7 \%$, respectively.

Data are expressed as means (and standard deviations) or percentages, and $p$ values below 0.05 were considered statistically significant. Age was categorized into 3 groups $(<55$, $55-64$ and $\geq 65$ years); the age- and sex-specific prevalences of lower-extremity amputation were calculated for type $\mathrm{I}$ and

Table 2: Prevalence of lower-extremity amputation by age and sex and by type of diabetes

\begin{tabular}{|c|c|c|c|}
\hline \multirow[b]{2}{*}{ Age, yr } & \multicolumn{2}{|c|}{ Group; \% (no.) of amputations } & \multirow[b]{2}{*}{$p$ value } \\
\hline & Men & Women & \\
\hline \multicolumn{4}{|c|}{ Type 1 diabetes } \\
\hline$<55$ & $1.0 \quad(7 / 668)$ & $0.3 \quad(2 / 603)$ & $>0.1$ \\
\hline $55-64$ & $2.8 \quad(10 / 354)$ & $2.9(12 / 419)$ & $>0.1$ \\
\hline$\geq 65$ & $1.6 \quad(9 / 550)$ & $2.1 \quad(14 / 665)$ & $>0.1$ \\
\hline All ages & $1.7 \quad(26 / 1572)$ & $1.7 \quad(28 / 1687)$ & $>0.1$ \\
\hline$p$ value & $>0.1$ & $<0.01$ & \\
\hline \multicolumn{4}{|c|}{ Type 2 diabetes } \\
\hline$<55$ & $0.6 \quad(64 / 11529)$ & $0.4 \quad(42 / 10078)$ & $>0.05$ \\
\hline $55-64$ & $0.8 \quad(94 / 11654)$ & $0.5 \quad(83 / 15$ 289) & $<0.01$ \\
\hline$\geq 65$ & $1.2(219 / 18215)$ & $1.0(228 / 23092)$ & $<0.05$ \\
\hline All ages & $0.9(377 / 41398)$ & 0.7 (353/48 459) & $<0.01$ \\
\hline$p$ value & $<0.01$ & $<0.01$ & \\
\hline
\end{tabular}


type 2 diabetes. The $\chi^{2}$ test was used to test differences in prevalence between sex and age groups.

Height was categorized into quartiles. Actual and adjusted prevalences of lower-extremity amputations were calculated for each quartile. Actual prevalences were tested by the linear test for trend. Covariates used to estimate the adjusted prevalences were age, sex, type of diabetes, duration of diabetes, smoking status and hypertension. ${ }^{14}$

Logistic regression analyses were used to estimate the odds ratios and 95\% confidence intervals for lower-extremity amputation for the various risk factors. Two models were created: the first was for all of the study subjects and adjusted for age, sex, type of diabetes, duration of diabetes, smoking status, hypertension and height. The second model was for the subgroup of patients who reported their fasting plasma glucose level and dyslipidemia status; these 2 factors were entered into the model in addition to the variables used in the first model.

\section{Results}

Of the 128572 randomly selected subjects, 93484 (72.7\%) completed the telephone survey. The age and sex distribution of the respondents was comparable to that of the original cohort of 256036 diabetic patients. ${ }^{11,12} \mathrm{~A}$ total of 368 patients were excluded because they were less than 18 years old, which left 93 II6 respondents; 9295 of them reported their fasting plasma glucose level and dyslipidemia status and were included in the subgroup analysis.

Table I shows the baseline characteristics of the respondents. A total of 784 patients (o.8\%) reported a history of lower-extremity amputation. The age- and sex-specific prevalences of such amputation by type of diabetes are shown in Table 2. The prevalence increased significantly with age among men and women with type 2 diabetes, but a significant increase with age was evident only among women with type I diabetes. Among patients with type 2 diabetes, men were significantly more likely than women to have had a lower-extremity amputation in all age groups except the youngest $(<55$ years).

Fig. I shows the actual and adjusted prevalences of lowerextremity amputation by height quartiles for all of the diabetic patients and for those with type I and type 2 diabetes. A significant increase in trends was observed $(p<0.05)$.

Fig. 2 shows the adjusted odds ratios for lower-extremity amputation among all of the subjects (model I) and among the subgroup of respondents for whom data were available on fasting plasma glucose level and dyslipidemia (model 2). In the first model, all of the factors except for smoking status (current smoker) and sex were found to be significant (Fig. 2, top panel). In the subgroup analysis (model 2), height, duration of diabetes, smoking status (ex-smoker), hypertension and fasting plasma glucose level were significant factors (Fig. 2, bottom panel).

\section{Interpretation}

This study revealed a strong and statistically significant association between height and risk of lower-extremity amputation among diabetic patients. The association held even after

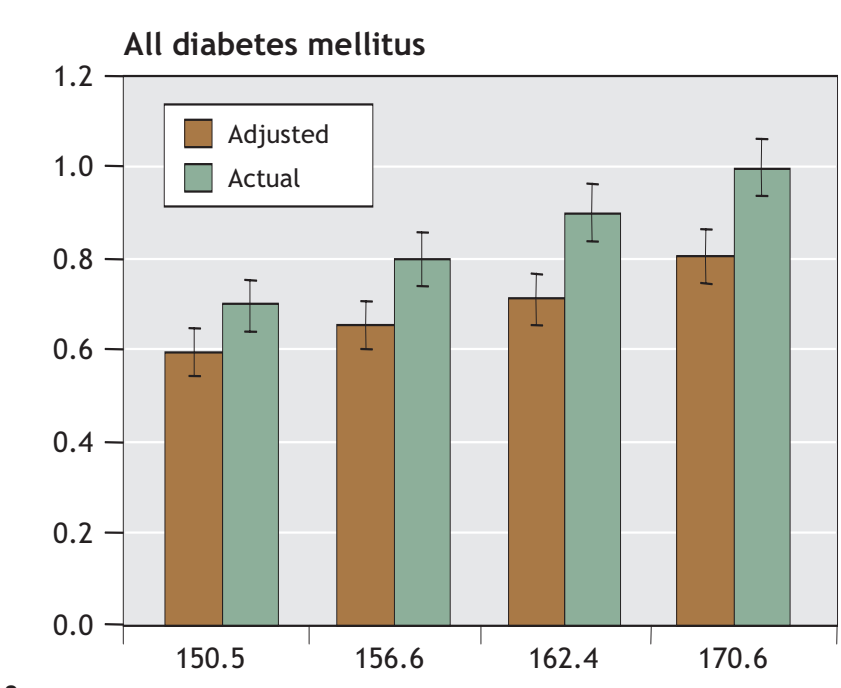

ภ̊

\section{Type 2 diabetes}
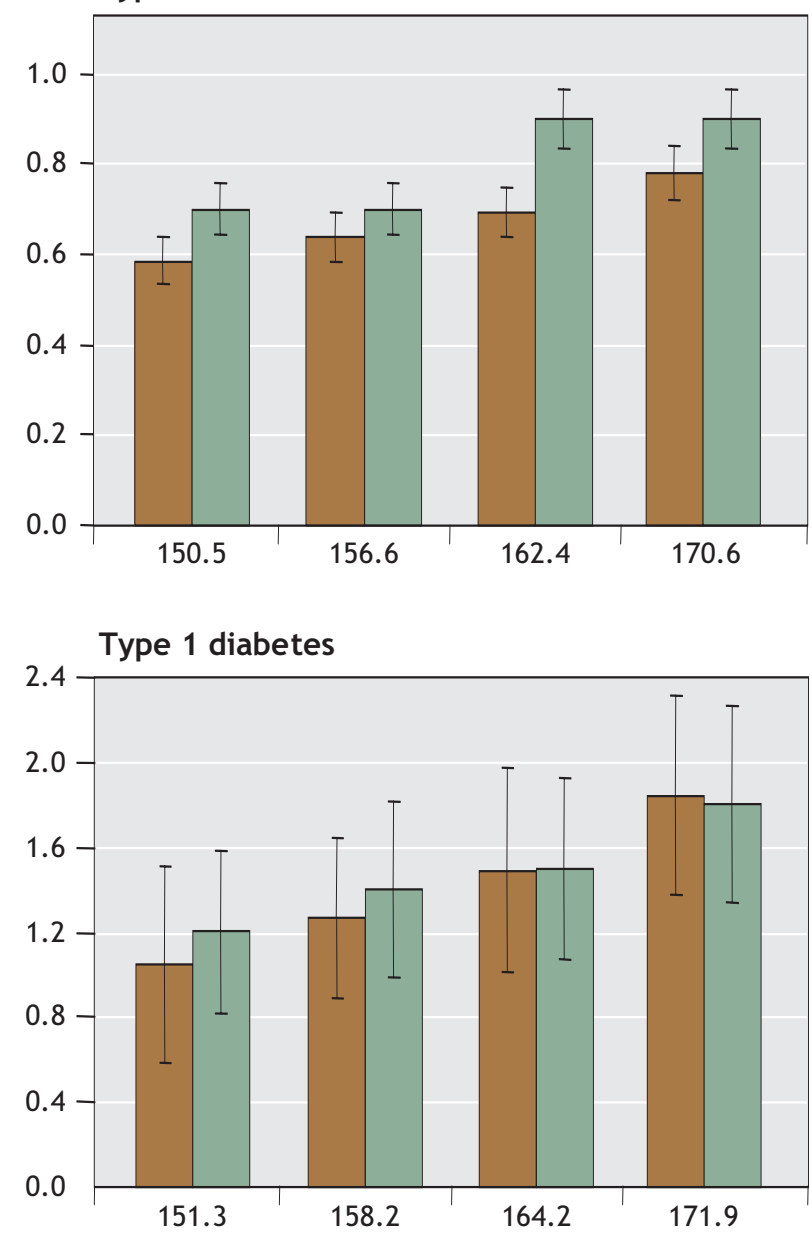

Height quartile, $\mathrm{cm}^{*}$

Fig. 1: Actual and adjusted prevalences of lower-extremity amputation by height quartile among patients with diabetes mellitus 18 years and older. Adjusted variables were age, sex, type of diabetes, duration of diabetes, smoking status and hypertension. Error bars $=$ standard errors. ${ }^{*}$ Values are mean heights for the respective quartiles. 


\section{Variable}

Type of diabetes (type 1 v. type 2)

Age (10-yr increment)

Sex (male v. female)

Duration of diabetes (10-yr increment)

Smoking status (ex-smoker v. never smoked)

Smoking status (current v. never smoked)

Hypertension (yes v. no)

Body height (10-cm increment)
Amputation : Amputation

less likely more likely

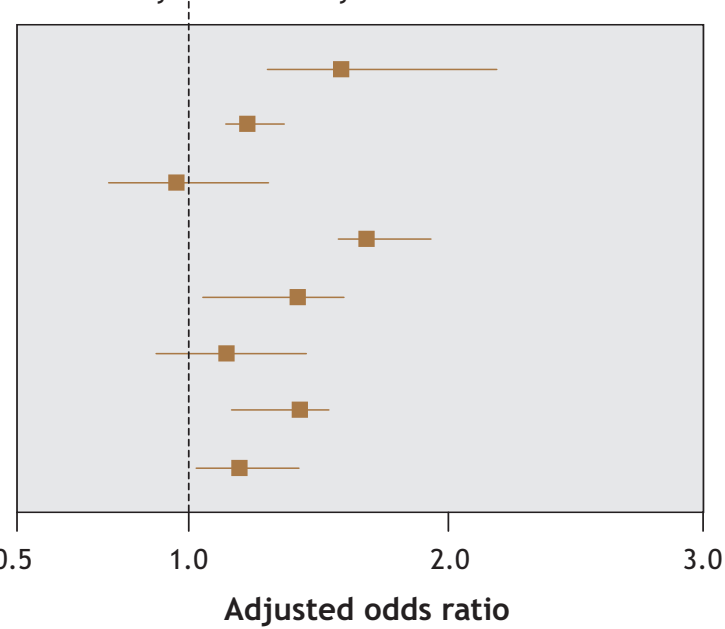

Adjusted OR (95\% Cl)

$1.67(1.24-2.25)^{*}$

$1.19(1.10-1.28)^{*}$

$0.98(0.77-1.24)$

$1.78(1.65-1.93)^{*}$

$1.33(1.05-1.69) \dagger$

$1.13(0.90-1.41)$

$1.34(1.15-1.57)^{*}$

$1.16(1.03-1.32) \dagger$

\section{Variable}

Type of diabetes (type $1 \mathrm{v}$. type 2)

Age (10-yr increment)

Sex (male v. female)

Duration of diabetes (10-yr increment)

Smoking status (ex-smoker v. never smoked)

Smoking status (current v. never smoked)

Hypertension (yes v. no)

Body height (10-cm increment)

FPG (0.6-mmol/L increment)

Dyslipidemia (yes v. no)

Dyslipidemia (unknown v. no)
Amputation : Amputation less likely more likely

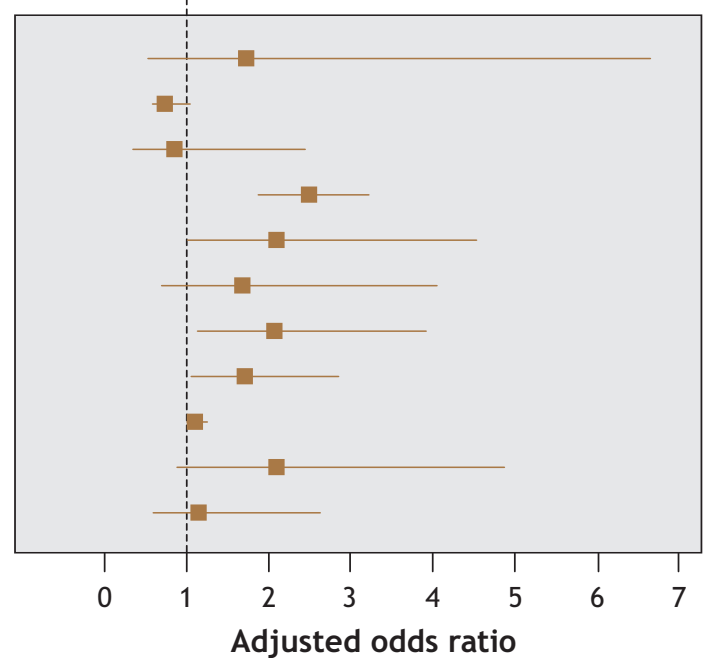

Adjusted OR (95\% Cl)

$1.83(0.50-6.65)$

$0.78(0.60-1.02)$

$1.00(0.40-2.45)$

$2.52(1.95-3.25)^{*}$

$2.18(1.03-4.58) \dagger$

$1.71(0.73-4.02)$

$2.18(1.20-3.96) \dagger$

$1.79(1.14-2.82) \dagger$

$1.12(1.04-1.21)^{*}$

$2.14(0.95-4.84)$

$1.27(0.61-2.63)$

Fig. 2: Multivariate-adjusted odds ratios (ORs) and $95 \%$ confidence intervals (Cls) for lower-extremity amputation among all 93116 diabetic patients (top panel) and among the 9295 patients for whom data of fasting plasma glucose (FPG) levels and history of dyslipidemia were available (bottom panel). ${ }^{\star} p<0.01 ; \uparrow p<0.05$.

adjustment for other risk factors for this type of amputation. Every additional $\mathrm{Io} \mathrm{cm}$ of height was associated with a $\mathrm{I} \%$ increase in the risk of amputation. In the subgroup analysis, in which adjustments were made for additional information on fasting plasma glucose level and dyslipidemia, the adjusted risk of amputation was even larger ( $79 \%$ for every additional Io $\mathrm{cm}$ of height).

This study had several limitations. Sensory dysfunction could not be evaluated. Taller patients are known to be at increased risk of peripheral sensory loss compared with shorter patients. ${ }^{1-7}$ The association between height and lowerextremity amputation would be underestimated if taller patients who had undergone amputation were less healthy and had more comorbidities (e.g., compression of vertebral disc space) than shorter patients who had undergone amputation.
The association between height and lower-extremity amputation was stronger in the subgroup analysis that adjusted for fasting plasma glucose level and dyslipidemia than in the main analysis that did not include these 2 factors. Because the data were collected over 7 years, a healthy survivor bias may have existed. If taller patients undergoing lower-extremity amputation are at lower risk of death than shorter patients undergoing such amputation, estimates of the effects of height on amputation might be overestimated. Follow-up of the cohort to determine the incidence of lower-extremity amputation would give a more assured answer.

The association between lower-extremity amputation and weight or body mass index (BMI) was not evaluated in this study because weight and BMI change significantly under different disease conditions and nutritional states and because 
the weight of lost limbs in amputated patients is difficult to estimate; height, on the other hand, changes minimally and will only decrease after puberty. The significant association between height and lower-extremity amputation persisted even after adjustments for weight and BMI (data not shown).

As in previous studies in Taiwan, ${ }^{9,11,15}$ diabetic men were found to be at higher risk of lower-extremity amputation than their female counterparts (Table 2). However, in the present study, sex did not remain a significant factor after adjustment for the other confounding variables (Fig. 2). In fact, in another logistic regression analysis in which only sex and height were included as independent variables, only height was found to be significantly associated with lower-extremity amputation (data not shown). This observation suggests that the difference in amputation prevalence between the men and women was due to a confounding effect of height.

In conclusion, height is a risk factor for lower-extremity amputation among diabetic patients. This effect appears to be independent of other known risk factors. Although the underlying mechanism could not be identified, it may be wise to pay particular attention to the early detection and treatment of leg ulcers in taller patients with diabetes.

\section{Editor's take}

- Peripheral sensory loss with foot ulcer in diabetic patients is the most common indication for lower-extremity amputation. Since taller diabetic patients are at greater risk of peripheral sensory loss than are shorter diabetic patients, are they also at greater risk of foot ulcers and subsequent amputation?

- In a survey of 93116 people in Taiwan with diabetes, height was found to be a strong predictor of lower-extremity amputation, even after adjustment for other known risk factors such as age and sex. In the whole study population, every $10-\mathrm{cm}$ increase in height was associated with a $16 \%$ increase in risk of amputation. In the subgroup of patients for whom data on fasting plasma glucose levels and dyslipidemia were available, the risk of amputation was even greater ( $79 \%$ for every $10-\mathrm{cm}$ increase in height).

Implications for practice: Taller patients with diabetes appear to be at increased risk of lower-extremity amputation and should be monitored closely for early signs of peripheral sensory loss.
This article has been peer reviewed.

From the Division of Endocrinology and Metabolism, Department of Internal Medicine, National Taiwan University Hospital; the National Taiwan University College of Medicine; the School of Public Health, Taipei Medical University; and the Division of Environmental Health and Occupational Medicine, National Health Research Institutes, Taipei, Taiwan

Competing interests: None declared.

Acknowledgements: The author thanks the Department of Medical Research, National Taiwan University Hospital, for providing the facilities, space and support for the related studies.

This study was supported in part by grants from the Department of Health (grant DOH89-TD-I035) and the National Science Council (grants NSC-90-2320-B-002-197, NSC-92-2320-B-002-156, NSC-93-2320-B-002-07I and NSC-94-2314-B-002-I42), Taiwan.

\section{REFERENCES}

I. Sosenko JM, Gadia MT, Fournier AM, et al. Body stature as a risk factor for diabetic sensory neuropathy. Am J Med ig86;80:103I-4.

2. Gadia MT, Natori N, Ramos LB, et al. Influence of height on quantitative sensory, nerve-conduction, and clinical indices of diabetic peripheral neuropathy. Diabetes Care 1987;10:613-6.

3. Sorensen L, Molyneaux L, Yue DK. Insensate versus painful diabetic neuropathy: the effects of height, gender, ethnicity and glycaemic control. Diabetes Res Clin Pract 2002;57:45-51.

4. Christen WG, Manson JE, Bubes V, et al. Risk factors for progression of distal symmetric polyneuropathy in type I diabetes mellitus. Sorbinil Retinopathy Trial Research Group. Am J Epidemiol I999;I50:II42-5I.

5. Adler AI, Boyko EJ, Ahroni JH, et al. Risk factors for diabetic peripheral sensory neuropathy. Results of the Seattle Prospective Diabetic Foot Study. Diabetes Care 1997;20:1162-7.

6. Maser RE, Laudadio C, DeCherney GS. The effects of age and diabetes mellitus on nerve function. J Am Geriatr Soc 1993;41:1202-4.

7. Robinson LR, Stolov WC, Rubner DE, et al. Height is an independent risk factor for neuropathy in diabetic men. Diabetes Res Clin Pract 1992;16:97-102.

8. Birke JA, Patout CA Jr, Foto JG. Factors associated with ulceration and amputation in the neuropathic foot. JOrthop Sports Phys Ther 2000;30:9I-7.

9. Tseng CH, Tai TY, Chen CJ, et al. Ten-year clinical analysis of diabetic leg amputees. J Formos Med Assoc I994;93:388-92.

Io. Global Lower Extremity Amputation Study Group. Epidemiology of lower extremity amputation in centres in Europe, North America and East Asia. BrJ Surg 2000; 87:328-37.

II. Tseng $\mathrm{CH}$. Mortality and causes of death in a national sample of diabetic patients in Taiwan. Diabetes Care 2004;27:1605-9.

I2. Tseng $\mathrm{CH}$, Chong CK, Sheu JJ, et al. Prevalence and risk factors for stroke in type 2 diabetic patients in Taiwan: a cross-sectional survey of a national sample by telephone interview. Diabet Med 2005;22:477-82.

13. Lin T, Chou P, Lai MS, et al. Direct costs-of-illness of patients with diabetes mellitus in Taiwan. Diabetes Res Clin Pract 200I;54(Suppl I):S43-6.

14. Lee J. Covariance adjustment of rates based on the multiple logistic regression model. JChronic Dis I981;34:415-26.

I5. Tseng $\mathrm{CH}$, Tai TY, Chong $\mathrm{CK}$, et al. Mortality in diabetic patients after lower extremity amputations. J Formos Med Assoc 1994;93:842-8.

Correspondence to: Dr. Chin-Hsiao Tseng, Department of Internal Medicine, National Taiwan University Hospital, No. 7 Chung-Shan South Rd., Taipei, Taiwan; fax 02 2937-8201;

ccktsh@ms6.hinet.net 